\title{
Mini Managers: Children strategically divide cognitive labor among col- laborators, but with a self-serving bias
}

\author{
Carolyn Baer \\ University of British Columbia \\ University of California, Berkeley
}

\author{
Darko Odic \\ University of British Columbia
}

\begin{abstract}
Strategic collaboration according to the law of comparative advantage involves dividing tasks based on the relative capabilities of group members. Three experiments $(\mathrm{N}=405$, primarily White and Asian, 45\% female, collected 2016-2019 in Canada) examined how this strategy develops in children when dividing cognitive labor. Children divided questions about numbers between two partners. By 7 years, children allocated difficult questions to the skilled partner (Experiment 1, $d=1.42$; Experiment 2, $d=$ 0.87). However, younger children demonstrated a self-serving bias, choosing the easiest questions for themselves. Only when engaging in a third-party collaborative task did 5-year-olds assign harder questions to the more skilled individual (Experiment $3, d=0.55$ ). These findings demonstrate early understanding of strategic collaboration subject to a self-serving bias.
\end{abstract}

Keywords: collaboration, cognitive labor, division of labor, skill, difficulty, law of comparative advantage

Supplemental Materials: https://osf.io/xeb6z/

Collaboration is a foundational feature of human culture and cognition, illustrated in part by its early emergence in childhood (Tomasello, 1999; Warneken, 2018). One of the challenging tasks when we engage in these collaborations is to strategically divide labor to achieve the team's goals rather than only our own. For instance, imagine yourself as a child in math class. Your teacher has just described today's activity: you and a partner must answer 100 math questions with a range of difficulties as fast as possible. But, while you are generally good at math, your partner is not. How do you best divide the questions to maximize the team's chance of success?

There are many possible solutions to this challenge. To maximize accuracy, you could answer all the ques-

Carolyn Baer, University of British Columbia and University of California, Berkeley; Darko Odic, University of British Columbia.

This work was funded by the Social Sciences and Humanities Research Council of Canada through an Insight Development Grant to DO and a Canada Graduate Scholarship to CB. Thanks to Natasha $\mathrm{Au}$, Bana Ashour, Nicaela Weigel, Nicole Gertz, Emilie Kniefel, Sally Poon, and Inderpreet Gill for their assistance with data collection, to CB's writing groups for feedback on portions of the manuscript, and to all the schools and families for their support.

Correspondence concerning this paper should be addressed to Carolyn Baer, Department of Psychology, University of California, Berkeley, 2121 Berkeley Way West, Berkeley, California, USA, 94720. Email: carolynbaer@berkeley.edu tions yourself. This would cost you considerable time and might hurt the feelings of your partner. To instead minimize time, you could divide the questions randomly into two equal piles. This might come at the cost of accuracy when your partner attempts the harder questions in their pile.

There is also a solution that optimizes both accuracy and time based on the economic law of comparative advantage (Ricardo, 1891). You could divide the questions in half by difficulty, assigning the easier questions to your partner and taking on the harder questions yourself. To illustrate, if you can answer $96 \%$ of easy questions correctly and your partner can answer $87 \%$, there is a $9 \%$ collective benefit if you answer the easy questions. But if you can answer $94 \%$ of hard questions correctly against $72 \%$ for your partner, the collective benefit is a much larger $24 \%$ if your partner answers the easy questions. (Estimates based on actual performance from Baer \& Odic (2019) Study 1 for 5- and 7-yearolds in a numerical comparison task like what we use in the present studies.) This means that the team gains $9 \%$ if you answer easy questions, but misses out on the much larger $24 \%$ benefit if you had answered the harder ones. Formally, you would have a 'comparative advantage' on the difficult questions because the benefit to the team is comparatively larger when you answer difficult instead of easy questions.

In everyday life, we employ economists to perform these computations on the scale of international trade 
and hire managers to make these decisions within organizations. But these decisions are not only made by highly-trained people; they also permeate everyday life. Groupwork must be divided among classmates, roommates must divide chores, and co-authors must decide which sections of the manuscript to write. These far-reaching uses of strategic thinking about skill and difficulty open a question of whether such strategic thinking might be fundamental to collaborative reasoning in humans. To examine this possibility, we investigate how strategic divisions of labor emerge in childhood.

\section{The Development of Strategic Division of Labor}

To understand when and how children reason about division of labor, we break down the formal computations needed to determine comparative advantage into several broad steps. We intend these to serve as an abstract set of minimal steps required for dividing labour rather than an exact model for how we carry them out. Strategists must estimate the chances of success on the given tasks for both parties. Those estimates need to be compared for their relative advantages. Finally, the resulting solution needs to be enacted. Current evidence suggests that each of these steps should be possible for even preschool children.

\section{Estimating the Chances of Success of Self and Other}

The literature on metacognitive reasoning demonstrates that preschool children can sensibly reason about their chances of success, or what is more commonly referred to as confidence (Pouget et al., 2016). Preschoolers report higher confidence on items they answer correctly than on items answered incorrectly (Hembacher \& Ghetti, 2014; Lyons \& Ghetti, 2011), demonstrating that can differentiate their likely success from likely failure. This reasoning also influences children's strategies, like decisions to ask for help, opt out of a task, or seek additional information (Balcomb \& Gerken, 2008; Call \& Carpenter, 2001; Coughlin et al., 2015; Goupil et al., 2016; Hembacher \& Ghetti, 2014).

Particularly relevant to our question here are recent findings that children strategically choose to answer items with higher chances of success. When given the option to select from a pair of perceptual quantity comparisons (e.g., which set has more dots, or which shape is bigger), 5-9-year-old children generally chose those featuring larger, easier ratios that they were more likely to answer correctly (Baer et al., 2021; Baer \& Odic,
2019). Children therefore seem able to estimate their individual relative chances of success and enact a simple strategy to maximize that success.

Young children can also reason about others' chances of success. Three-year-olds recognize and strategically seek help from individuals who have been previously accurate or have relevant expertise, inferring that they will continue to be informative in the future (Birch et al., 2008; Harris et al., 2018; Koenig \& Harris, 2005; Lutz \& Keil, 2002; Mills, 2013; Pasquini et al., 2007). By 4 years, children tailor their communication based on the linguistic skill and world knowledge of others (Baer \& Friedman, 2018; Shatz \& Gelman, 1973), using fewer words and talking about general rather than specific properties to ensure successful understanding. Further, preschoolers opt to help someone faced with a difficult task rather than an easy one, reflecting an understanding that the chances of success were lower on the difficult task (Bennett-Pierre et al., 2018; Bridgers et al., 2020; Ronfard \& Harris, 2017). Children therefore demonstrate their understanding of others' chances of success for achieving their own goals and when assisting others achieve goals.

\section{Formulating and Enacting a Solution}

Collaboration involves working towards a group goal rather than individual goals. While the optimal strategy for an individual may be to prioritize easier work and seek help on difficult work, the law of comparative advantage shows that a skilled team member always pursuing the easiest option can negatively impact the team's collective chance of success. Collaboration therefore requires thinking about and balancing both one's own and another's chances of success.

Two studies provide initial evidence that children consider both parties' capabilities in collaborative settings. Warneken and colleagues (2014) had pairs of 35 -year-old children tasked with retrieving a reward using two complementary tools. After viewing their partner choose one of the tools, 5-year-olds consistently chose the other tool to ensure that the team had access to both. Three-year-olds also made this strategic choice, but only if their partner's choice was initially constrained to only one of the tools (Warneken et al., 2014). This behavior reflects an emerging understanding that success as a group depends on the combined and complementary capabilities of the team members.

Another study by Magid and colleagues (2018) suggests that children can further apply this collaborative strategizing to the skill of each partner in dealing with 
physical constraints. In their study, 4-year-olds needed to win two one-shot carnival games that required throwing objects at targets either close or far away. The games needed to be won simultaneously, so children were given a partner and asked to assign each person one game to play. If their partner was older than them (with presumably better chances of success), children assigned the harder game to the partner and the easier game to themselves. In contrast, children assigned the harder game to themselves if their partner was younger (Magid et al., 2018). This strategy, consistent with the law of comparative advantage, suggests that even preschool children can consider the relative chances of each partner successfully overcoming the physical constraints of the tasks.

\section{Strategic Division of Cognitive Labor}

When these findings are put together, there is good reason to believe that children as young as 4 years old can follow the law of comparative advantage when making strategic collaborative decisions. In this work, we test whether this strategy extends to a non-physical domain, as would be expected if it was of prime importance for human collaboration.

Human collaboration spans many different domains from physical (e.g., lifting a couch together) to emotional labor (e.g., caring for elders). In particular, many of the everyday tasks that require dividing labor are not subject to physical constraints like the distance of targets, but by cognitive constraints like knowledge, memory, or mental effort. In our math class example, some group members may not have the knowledge required to answer harder questions at all - a constraint on their performance that is determined by the effort and ability required entirely of the $\neg$ mind, not the body. With the evidence that children can estimate their own and others' success on similar knowledge-based tasks (e.g., Baer \& Friedman, 2018; Baer \& Odic, 2019), it seems highly likely that children would similarly use the law of comparative advantage when strategizing about tasks from this cognitive domain. Given this possibility, along with the importance of cognitive labor for engaging in modern society, we therefore focus on collaboration in cognitive labor here.

At the same time, strategically dividing cognitive labor may be more difficult than dividing physical labor for young children because of potential differences at each of the three theorized steps outlined above. First, children could have more difficulty reasoning about another's chances of success at tasks requiring cognitive skill than physical skill (Niebaum \& Munakata, 2020).
Cognitive skills like knowledge or mental capacity have few, if any, concrete correlates available prior to observing their success or failure. In contrast, many physical skills can be inferred from appearances only. For example, we don't expect infants to be capable of running, but we do expect people with large muscles to be able to lift heavy weights. It could therefore be that children can learn about physical skills more readily because there are concrete correlates in the world, while cognitive skill understanding may lag behind given its more abstract nature. If so, children may need more time and experience to learn about these abstract relations, or because they conceptualize cognitive skill as linked to a concrete property (like age or wearing glasses). The current literature has little to say about this, as there is currently a lack of evidence about whether children reason differently about cognitive and physical skills.

A second possibility is that the law of comparative advantage strategy must be re-learned for each applicable domain. Children do not always apply strategies learned in one domain to others (Bellon et al., 2020; Geurten et al., 2018; Vo et al., 2014). For instance, children who strategically placed high bets on easier number discriminations did not necessarily bet high on easier emotion discriminations (Vo et al., 2014; see also Bellon et al., 2020; Geurten et al., 2018). Therefore, even though Magid and colleagues (2018) found performance consistent with the law of comparative advantage in young children within the physical domain, children might need to re-learn this strategy separately for other domains.

A final possibility considered here is that children may be subject to stronger task biases that overshadow the appropriate strategy when executing their strategy to divide cognitive labor. One such bias is well-documented in the literature on children's understanding of fairness (Blake, 2018; Blake et al., 2014). Children up to 6-8 years show a self-serving bias, prioritizing their own self-interest over others' by giving themselves more stickers than a partner (Blake et al., 2015; Blake $\&$ McAuliffe, 2011; Sheskin et al., 2016). At the same time, children this age and even preschoolers will identify these biased distributions as unfair when made between two other parties (Chernyak \& Sobel, 2015; Rochat et al., 2009; Sheskin et al., 2016). Children thus possess knowledge of the 'correct' option but it is overshadowed by other influences on their behavior, like a desire to give themselves an advantage (Blake, 2018). A similar phenomenon may exist here, where children can formulate a strategy according to the law of comparative advantage, but their behavior is dominated by 
a self-serving bias. This bias could differentially affect the division of cognitive labor if intellectual competence is more highly prized by children than physical competence. There is again little evidence in the current literature to speak to this (though see Asaba \& Gweon, 2019; Zhao et al., 2018 for evidence that children are motivated to appear competent).

\section{The Current Studies}

In our studies, we ask whether children apply the law of comparative advantage to their strategic division of cognitive labor. We presented children with a task relying on cognitive skill: intuitive number comparisons ("which one has more dots"; Halberda \& Feigenson, 2008). Children divided the questions between themselves and a peer described as being 'better' or 'not as good' at the task. Children strategically assigned themselves the easier questions when their partner was 'better' and likely more capable of succeeding on the difficult items. However, to anticipate the findings of our first experiment, we did not find that children made strategic divisions of cognitive labor until much older, at age 9 .

In two follow-up studies, we investigate two of the three possible difficulties as explanations for why children did not appear to follow the law of comparative advantage when dividing cognitive labor: difficulty estimating the chances of success and difficulty in enacting a solution. If children fail to use this strategy across all studies, even with modifications to account for these two difficulties, we would instead have initial evidence that children struggle to formulate the strategy when reasoning about cognitive labor.

\section{Experiment 1}

\section{Method}

Children were made to believe that they would play a collaborative "Number Game" (described below) with another child, who was either better or worse at that game than the child. We then offered children the opportunity to divide questions from the Number Game between the two partners to maximize their chances of success. Of critical interest was whether children would assign the easier of the two questions to the relatively less skilled group member, consistent with the law of comparative advantage. That is, would children assign easier questions to themselves if they were less skilled, but assign those same easy questions to their partner if the partner was less skilled?

\section{Participants}

One-hundred and fifty-nine children participated in the study (81 girls) between March 2016 and March 2017. Children were between the ages of 6 and 10 years old $(\mathrm{M}=7 ; 11$ [years; months], range $=6 ; 0-9 ; 11)$ as this age group is known to attend to and strategically compare cognitive difficulty in the number discrimination task we used (Baer et al., 2018; Baer \& Odic, 2019). Our target sample size, set prior to testing based on the recommendations of Simmons et al., (2011) though not formally preregistered, was 20 children in each condition at each age (160 total). Most children participated in a quiet area of their school and some participated in an on-campus lab, all in Vancouver, British Columbia, Canada. We did not formally collect demographic information, but children largely matched the demographics of the region (predominantly middleclass, and White or East or South-East Asian), and all children spoke English. Two additional children were excluded for having known or suspected developmental disabilities (reported by the child's classroom teacher). Informed consent was given by children's parents prior to beginning the study.

\section{Materials and Procedures}

Tasks were presented on a laptop computer using Psychtoolbox-3 in MATLAB (Brainard, 1997; The MathWorks Inc., 2015). Children responded verbally or by pointing to their answer on the screen. The experimenter entered all responses to reduce the influence of memory and motor development on the results.

Number Game. In each trial, children selected which of two groups of dots is more numerous without counting (e.g., 10 yellow dots is more than 5 blue dots, see Figure 1). As mentioned earlier, smaller ratios, like 8 yellow to 9 blue (a ratio of 1.13), result in longer decision times and lower accuracy than large ratios, like 15 yellow to 5 blue (ratio 3.0; Baer \& Odic, 2019; Halberda \& Feigenson, 2008). These reaction time and accuracy differences signal that smaller ratios are more difficult and require more cognitive skill than larger ratios. By 5 years of age, children are sensitive to these differences in difficulty and can strategically identify the easier of two ratios (Baer \& Odic, 2019, 2020). Therefore, we manipulated the difficulty of each trial through the ratio of dots, from a hard 1.13 to an easy 3.0. 
a) The Number Game

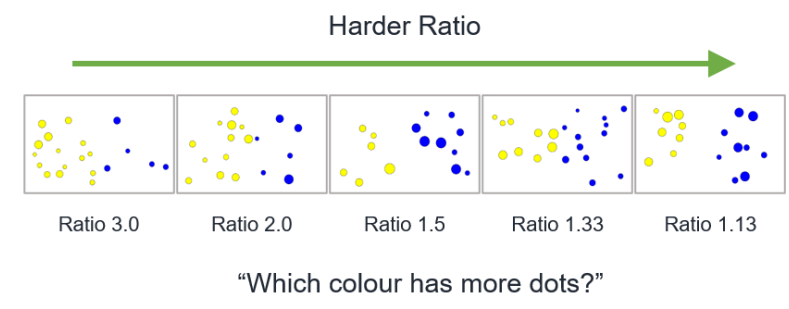

b) Collaborative Task (Allocation)

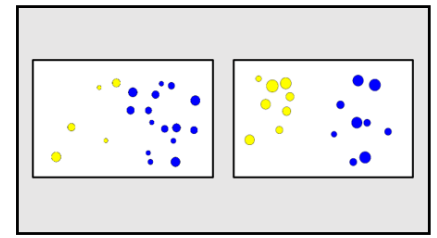

"Pick one for you and one for Sam" (Exp. 1 \& 2)

"Pick one for Sam and one for Jesse" (Exp. 3)

Figure 1. Stimuli Used in the Experiments. (a) shows sample trials from the Number Game at different ratios. Children indicate which color has more dots. Larger ratios (on the left) are easier and smaller ratios (on the right) are more difficult. (b) shows a sample trial from the collaborative task. Children assign each question to a group member. In this example, the easier question is on the left.

Children were first introduced to the Number Game through 10 warm-up trials. On each trial, the dots appeared on the screen for $1.2 \mathrm{sec}$, and the child indicated whether the yellow or blue set was more numerous. The computer played audio feedback about the accuracy of each trial (e.g., "Oh, that's not right." or "Great!"). Because our collaborative task (detailed below) sometimes required that children believe they were less skilled than their partner, two of the ten warm-up trials were impossible (e.g., 10 yellow and 10 blue dots) and always led to negative feedback. These impossible trials served to signal to children that they were not perfectly skilled at the game, making it plausible that another child could be more skilled than them. The remaining 8 trials were relatively easy ratios (2.0 and 3.0) to get children comfortable with the task.

Collaborative Task. Children were given a simple collaborative goal: they and a partner both needed to answer a lot of Number Game questions correctly to win the game. Each partner would answer half of the total questions independently, and the two independent scores would be combined to form a team score. The exact number of correct answers needed was not provided as we did not want children to begin counting the number of trials, or feel too anxious about getting an answer wrong. If children asked for a specific number (which happened very rarely), the experimenter told them that she didn't know the exact number, but emphasized that it was 'a lot' to make clear that both the child and their partner would need to do well in order to win. Teams consisted of two children: the child participant and a virtual gender-matched 'partner,' introduced through a photo of a child (approximately 7 years old and White) on the computer screen. Partners were said to be in another room or at another school, but connected virtually. In the rare event a child expressed doubt about the partner's existence, the experimenter explained that the computers were connected (through FaceTime or Skype), and pretended to message 'the other experimenter' through an iPad.

We then provided children with information about the relative skill levels of each partner. Half of children were told that their partner answered more of the warmup questions correctly and was therefore better at the Number Game than the child ("Partner Better" condition, exact numbers were not provided). The other half heard that the partner didn't answer as many questions correctly and was therefore not as good at the Number Game ("Partner Worse" condition, randomly assigned). The experimenter also provided affective cues to differentiate the conditions (e.g., excited expression and tone if the partner did better, as though children were lucky to have a skilled partner, or a worried expression and tone if the partner did worse). The experimenter asked children to indicate which person was better at the game and corrected them before moving on if they answered incorrectly or did not want to answer.

As the focal task, we asked children to split pairs of Number Game questions between the partners. Our primary dependent variable was how often children would assign the easier question to themselves, expecting this to occur more frequently in the Partner Better condition than in the Partner Worse condition if the children are following the law of comparative advantage (e.g., Magid et al., 2018). In 14 test trials, children saw a pair of Number Game questions that differed in difficulty and were asked to choose one for their partner to answer and one for themselves to answer (see Figure 1 and see Baer \& Odic, 2019 for a similar design in a nonsocial task). If children only selected one question, the experimenter asked children to select which person would answer that question, and then indicated that the remaining question would go to the other partner, to make sure that children understood that they needed to assign both questions. Number Game questions varied in ratio from 1.13 (hard) to 3.0 (easy) and were paired 
to make test trials with 'metaratios' from 1.33 (small difference in difficulty, e.g., ratios 1.5 and 1.13) to 2.65 (large difference in difficulty; see Baer \& Odic, 2019). Children saw two trials at the easiest metaratio first, then cycled through the full range of metaratios in a random order that was the same for all children. We expected children's performance to be best on the largest metaratios, but additional metaratios were included to match the stimuli from Baer \& Odic, 2019 and to control for non-numeric cues on these trials (e.g., the cumulative area of the dots). We made no further predictions about this variable. The task was untimed, but children were prevented from counting the dots. Questions were never labelled as 'easy' or 'hard;' children had to infer these difficulties on their own. No feedback was provided to children about their choices at any point during the task. While splitting the trials, children were not asked to answer the questions, and therefore did not get any feedback about their performance on those trials.

Following the division of questions, children answered 14 Number Game questions. All children answered the same easy 14 questions, but were told that they were answering the questions they chose earlier. At the end of the session, children were told that their team had answered enough questions correctly to win and were given a small prize.

\section{Results}

All analyses below collapse across gender, as there was no impact of gender on these results.

First, we confirmed that children understood the Number Game using the 14 questions presented following the division of labor. As shown in Table 1, children at all ages clearly understood this task, as they selected the more numerous set well above chance of $50 \%$.

Given that children understood the Number Game, we next examined their division of labor to see whether they strategically allocated questions according to the law of comparative advantage. We conducted a 2 (Condition, between subjects: Partner Better, Partner Worse) by 4 (Age Group, between subjects: 6, 7, 8, 9) by 6 (Metaratio, within subjects: $1.33,1.5,1.77,2.0$, $2.26,2.65)$ ANOVA on children's choice to assign the easier question to themselves, averaged across the trials. The analysis of Condition was meant to confirm prior work (Magid et al., 2018), while Age Group and Metaratio were exploratory variables.
Table 1

\section{Means and Tests Against Chance in Experiment 1}

\begin{tabular}{ccccccc}
\hline $\begin{array}{l}\text { Age } \\
\text { (Years) }\end{array}$ & $\begin{array}{l}\text { Mean } \\
(\%)\end{array}$ & SD & $d f$ & $t$ & $p$ & $d$ \\
\hline \multicolumn{7}{c}{ Number Game (chose more numerous) } \\
6 \\
6 & 85.26 & 12.60 & 38 & 17.47 & $<.001$ & 2.80 \\
7 & 86.67 & 11.75 & 39 & 19.73 & $<.001$ & 3.12 \\
8 & 86.99 & 10.38 & 40 & 22.81 & $<.001$ & 3.56 \\
9 & 89.32 & 9.36 & 38 & 26.24 & $<.001$ & 4.20 \\
7 & & & & & \\
Division & (gave easier to self) & & & & \\
Partner Better Condition & & & & \\
6 & 61.56 & 16.21 & 20 & 3.27 & .004 & 0.71 \\
7 & 62.50 & 20.71 & 19 & 2.70 & .014 & 0.60 \\
8 & 61.07 & 24.90 & 19 & 1.99 & .061 & 0.44 \\
9 & 73.02 & 18.26 & 17 & 2.64 & .017 & 1.26 \\
Partner Worse Condition & & & & \\
6 & 60.71 & 17.19 & 17 & 2.64 & .017 & 0.62 \\
7 & 57.86 & 26.00 & 19 & 1.35 & .192 & 0.30 \\
8 & 45.92 & 26.78 & 20 & -0.70 & .493 & 0.15 \\
9 & 42.52 & 23.20 & 20 & -1.48 & .155 & 0.32 \\
\hline
\end{tabular}

Overall, children's strategies were consistent with the law of comparative advantage: they were more likely to take the easier question when they were less skilled than their partner than when they were better, $F(1,151)=10.65, p=.001, \eta_{\mathrm{p}}{ }^{2}=.07$. However, this effect was modulated by age, $F(3,151)=3.39, p=.020$, $\eta_{\mathrm{p}}{ }^{2}=.06$. As shown in Figure 2, a Tukey posthoc analysis revealed that only 9-year-olds matched skill and difficulty, keeping many more difficult trials for themselves in the Partner Worse condition than in the Partner Better condition, $t(37)=30.50, p<.001, d=1.46$. The conditions did not differ from one another in any other age group, though, as can be seen in Table 1 and Figure 2, 8-year-olds showed the same differentiation as the 9-year-olds but did not reach traditional levels of significance.

We also found a main effect of Metaratio (the ratio of difficulties being compared on each division trial), $F(4.4,663.75)=4.65, p=.001, \eta_{\mathrm{p}}^{2}=.03$ (GreenhouseGeisser corrected for sphericity). Similar to when children compare difficulties to make strategic choices only for themselves, children were more likely to choose the easier question when the difference in difficulty was large than when is was small (Baer et al., 2018; Baer \& Odic, 2019). There were no other significant main effects or interactions. We also repeated these analyses in the Supplemental Materials treating age as a continuous variable rather than categorial, and 


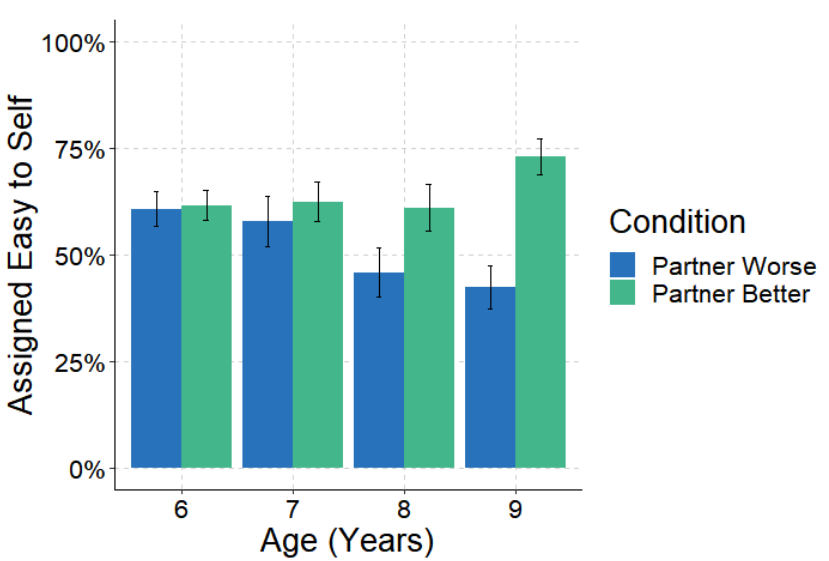

Figure 2. Percentage of Trials Assigning the Easier Question to Self in Experiment 1. Error bars represent 1 standard error.

looking only at performance on the first trial. Both analyses replicate the patterns reported here.

Finally, children generally allocated the easier question to themselves across both conditions. As shown in Table 1 and Figure 2, children at all ages took the easier question above chance of $50 \%$ when their partner was better, as expected given a strategy of matching skill with difficulty. However, when their partner was worse, 6-year-olds still took the easier question above chance - opposite the matching strategy - and 7-9year-olds' selections did not differ from chance.

\section{Discussion}

Only 9-year-olds in the current study strategically divided cognitive labor according to the law of comparative advantage. This is surprising given evidence that 4-year-olds enacted this strategy for a physical task (Magid et al., 2018) and that 5-year-olds can reason about the relative difficulties of questions in the identical Number Game (Baer \& Odic, 2019). Moreover, children in all age groups appeared generally biased to allocate the easier question to themselves, even when their partner was worse at the game, suggesting that they did recognize their own chances of success were higher on the easier questions than the harder ones.

If children could detect the differences in difficulties, then perhaps the challenge lies in their understanding of the skill manipulation. In a follow-up experiment in the Supplemental Material, we rule out other lowlevel explanations for not understanding the skill manipulation through a post-test comprehension check. We found that $91 \%$ of children passed the check yet still took easier questions for themselves. Therefore, properties of the design like the small number of warmup trials from which to form ability beliefs, the reliance on verbal testimony about partner performance, or forgetting over the course of the study are unlikely to account for the results.

Our initial intuition was that the term "better" should have directly cued skill without requiring any inference, and was therefore the best choice of skill manipulation. However, 4-year-olds succeeded at dividing physical labor in the studies by Magid et al., (2018) when cued to skill using the relative age of the partner ("younger" or "older"), which we learned about after completing this experiment. This highlights an intriguing possibility: perhaps as children learn about others around them, they may find it easier to link success with concrete, observable traits than these abstract ones. Therefore, children may have encoded the term "better", but not fully understood its implications for the underlying cognitive skills. In our next study, therefore, we used age as a more easily observable trait that correlates strongly with many cognitive skills like those required here (Halberda \& Feigenson, 2008; and see Magid et al., 2018). We told children that their partner was either older or younger than they were, accompanied by a picture of an older or younger child, respectively. We hypothesized that age alone might be a more salient and relevant cue to cognitive ability for children than direct testimony about skill, and that children might use this information to strategically divide cognitive labor younger than age 9 .

\section{Experiment 2}

\section{Method}

Participants. One hundred and sixty-six children participated in the study (74 girls) between February and July 2019 in the same manner and geographic location as Experiment 1. Our target sample size, as in Experiment 1, was 20 children per condition per age group (160 total). We recruited children between the ages of 4 to 8 years $(M=5 ; 11$, range $=4 ; 0-7 ; 11)$ because our goal was to detect success in children younger than age 9 and because the manipulation of age was successfully used by Magid and colleagues (2018) in 4-year-old children. Four additional children were excluded from the analyses for not competing the study (2), not understanding English (1), and because they participated in Experiment 1 (1).

Materials and Procedures. We used the same design as Experiment 1 with a few key changes. First, we did not tell children anything about the relative skill or 
past success of their partner, but instead told children that their partner was older or younger than them. To help reinforce this, the picture of their virtual partner was either about 2-3 years old if younger, or around 78 years old if older, ensuring that every age tested would believe the manipulation. Second, we provided a longer warm-up phase, including 30 trials ranging in ratio from 1.13 to 3.0. Like in Experiment 1, we included 6 impossible trials so that children did not get all questions correct. Third, we used more distinct metaratios $(2.0,2.88$, and 3.66) during the collaboration phase of the study to make sure that children could consistently tell apart the two difficulties. Fourth, we asked children two post-test comprehension questions to confirm that they remembered the key age manipulation ("Who is older?") and to see if they explicitly mapped age on to skill ("Who is better at the [Number] Game?").

\section{Results}

Children's gender is collapsed in these analyses, as its inclusion did not influence any of the results below. Gender interacted with metaratio, $\mathrm{F}(1.84,275.98)=$ $3.77, \mathrm{p}=.027, \eta \mathrm{p} 2=0.03$ (Greenhouse-Geisser corrected), but as neither are key variables of interest and do not interact with the condition manipulation, we did not look at this further.

First, we examined children's post-test comprehension answers to confirm that the skill manipulation worked. When asked which partner was older, 97.6\% of children gave the correct answer. When instead asked which partner was better at the Number Game, 109 children $(65.7 \%)$ responded with the older partner. Interestingly, an exploratory logistic regression revealed that this was influenced by condition (Odds Ratio $\left.(\mathrm{OR})=4.73, \chi^{2}(1)=17.40, \mathrm{p}<.001\right)$, age $(\mathrm{OR}=$ $2.19, \chi 2(1)=10.10, \mathrm{p}=.001)$, and their interaction (OR $=6.6, \chi 2(1)=6.60, p=.010)$. Simple slopes revealed that there was no age effect for linking age to skill if their partner was younger $(81.7 \%$ of children linked the two), $\beta=-0.20, \mathrm{SE}=0.29, \mathrm{t}(162)=-0.68, \mathrm{p}=.494$, but a strong age effect if their partner was older, $\beta=0.79$, $\mathrm{SE}=0.25, \mathrm{t}(162)=3.17, \mathrm{p}=.002($ from $36.3 \%$ at age 4 to $85.0 \%$ at age 7 ). We make two conclusions from this. First, children remembered the age manipulation and generally linked it to skill. Second, children, particularly younger children, were reluctant to label themselves as 'worse' at the task. No results below change when removing children who failed the comprehension check.
We next checked that children understood the Number Game. As shown in Table 2, children selected the more numerous color more often than $50 \%$ (chance) at all ages tested.

Table 2

Means and Tests Against Chance in Experiment 2

\begin{tabular}{cllllll}
\hline Age & Mean & SD & df & $t$ & $p$ & $d$ \\
\hline \multicolumn{7}{c}{ Number Game } \\
\multicolumn{4}{c}{ (chose more numerous) } \\
5 & 71.87 & 12.98 & 40 & 10.79 & $<.001$ & 1.69 \\
5 & 80.38 & 8.04 & 43 & 25.05 & $<.001$ & 3.78 \\
6 & 84.39 & 11.72 & 43 & 19.47 & $<.001$ & 2.93 \\
7 & 89.28 & 6.34 & 36 & 37.68 & $<.001$ & 6.19
\end{tabular}

Division (gave easier to self)

Partner Older Condition

\begin{tabular}{rllllll}
4 & 45.13 & 14.69 & 21 & -1.56 & .135 & 0.33 \\
5 & 49.03 & 16.83 & 21 & -0.27 & .789 & 0.06 \\
6 & 56.07 & 21.53 & 19 & 1.26 & .222 & 0.28 \\
7 & 65.00 & 24.84 & 19 & 2.70 & .014 & 0.60 \\
\multicolumn{6}{c}{ Partner Younger Condition } \\
4 & 46.24 & 6.89 & 18 & -2.38 & .029 & 0.55 \\
5 & 39.94 & 17.45 & 21 & -2.71 & .013 & 0.58 \\
6 & 51.19 & 25.33 & 23 & 0.23 & .820 & 0.05 \\
7 & 44.96 & 20.94 & 16 & -0.99 & .336 & 0.24 \\
\hline
\end{tabular}

Did children strategically assign the difficult questions to the older partner? We conducted an ANOVA with Condition (Partner Older, Partner Younger), Age $(4,5,6,7)$, and Metaratio $(2.0,2.8,3.66)$ on children's choice to assign themselves the easier question. Children were generally more likely to take the easy question when their partner was older than when their partner was younger, $\mathrm{F}(1,158)=6.42, \mathrm{p}=.012, \eta \mathrm{p} 2=.04$. Children's age also affected their behavior: older children in the sample were also more likely to take the easy question than younger children, $\mathrm{F}(3,158)=2.92$, $\mathrm{p}=.036, \eta \mathrm{p} 2=.05$. While not reaching conventional levels of significance, the analyses and Figure 3 hint at an interaction between the Condition and Age, F(3, $158)=2.14, p=.097, \eta p 2=.04$. Post-hoc Tukey tests revealed that only 7 -year-olds changed their division of labor strategy based on the relative age of their partner, $t(35)=-20.04, p=.044, d=0.87$, shown in Figure 3 . This appears to be a much clearer difference between conditions compared to 7-year-olds in Experiment 1, but also a more tenuous success than that of older children given the non-significant interaction term here, and so should be interpreted with caution. There was no main effect nor any interactions with Metaratio. 
These results also emerge when treating age as a continuous variable and when only examining the first trial (see Supplemental Materials).

As shown in Table 2, children were not biased to assign themselves the easier question, and instead generally divided question difficulty at chance levels.

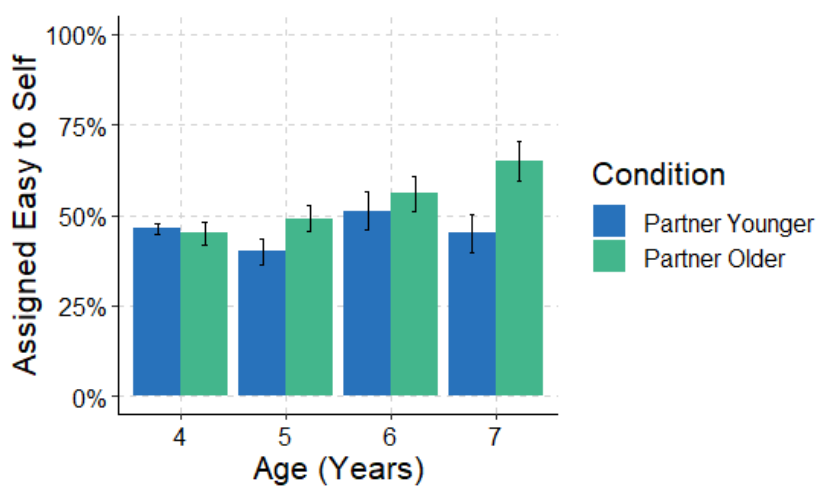

Figure 3. Percentage of Trials Assigning the Easier Question to Self in Experiment 2. Error bars represent 1 standard error.

\section{Discussion}

In contrast to Experiment 1, we have preliminary evidence that 7-year-olds strategically assigned easier questions to the relatively less skilled (i.e., younger) partner when age was used as a proxy for skill. As an easily observable trait in childhood, and one that often covaries with cognitive ability given the dramatic cognitive development in this age range, children may more readily link age with cognitive skill than the comparative testimony used in Experiment 1 . These results suggest that at least some of the apparent failure to strategically divide cognitive labor in 5-8-year-old children might stem from the labelling of skill, especially for the youngest children.

The results of Experiment 2 also highlight another, non-mutually exclusive possibility: children in Experiment 1 may have had a self-serving bias, choosing to act in their own self-interest rather than in the best interests of the team. Recall that in Experiment 1, children on average assigned themselves the easier question in both conditions. This is the same behavior seen when children try to maximize their own success, shown when children divided labor for a competition (Magid et al., 2018), and in an asocial setting (Baer et al., 2018; Baer \& Odic, 2019). In Experiment 2, however, children did not show this general preference for easy trials, instead assigning themselves the easier question about half of the time, independent of their partner's skill or age. We suspect that the key reason for this change is in the overt skill comparison used in
Experiment 1 ("Sam was even better than you"), which tends to lead to reward-maximizing behavior in schoolaged children. For instance, preschool children who were told they had a reputation for being smart were more likely to cheat (Zhao et al., 2018), and children who were outperformed by peers persisted longer than when peers performed worse (Magid \& Schulz, 2015). In contrast, children in Experiment 2 only learned about the relative age of the partner, which potentially spared them from these competitive feelings. In fact, we only observed similar self-preservation behavior when we asked children to compare which partner was better as part of our comprehension check at the end of the study. Children who were younger than their partner were more likely to respond that they were better or simply refuse to answer. Even if children knew they were younger, they seem to have felt uncomfortable saying they were worse at the game. If true, children's selfserving biases might mask their underlying understanding of strategic division of cognitive labor.

A similar kind of self-serving bias can be seen in the literature on children's fairness (e.g., Blake et al., 2015), where children are tasked with dividing resources like stickers or candies between partners. When presented with a split that gives them an advantage (e.g., they get 4 candies when their partner gets 1), children up to middle childhood (around age 8 ) generally accept the split (Blake et al., 2015; Blake \& McAuliffe, 2011; Sheskin et al., 2016). When instead presented with a split that unfairly advantages their partner (e.g., gives the partner 4 candies when they only get 1 ), children generally reject the split, leaving both partners with no resources. However, even preschool children say that it is only fair when the two partners receive equal resources, resulting in a gap between their knowledge of fair distributions and their actual distribution behavior (Blake, 2018; Blake et al., 2014). To combat this discrepancy and capture children's understanding of fairness using a behavioral measure, researchers rely on a third-party task, where children divide resources between two other parties rather than between themselves and a partner. When dividing resources between others, even preschool children divide resources equally (Chernyak \& Sobel, 2015; Rochat et al., 2009; Sheskin et al., 2016).

To understand the contribution of a self-serving bias in children's collaborative behavior, we used a thirdparty design in the next study. Rather than asking children to divide questions between themself and a partner, we asked children to divide questions between two other children that differ in skill (but are of same age). 
In doing so, we eliminate any potential self-serving biases, allowing us to directly test whether children younger than 7 will strategically divide cognitive labor according to the law of comparative advantage.

\section{Experiment 3}

\section{Method}

Participants. Eighty children between the ages of 4 to 8 years $(M=6 ; 0$, range $=4 ; 0-7 ; 11,29$ girls $)$ participated in the study between November 2016 and March 2017 in the same manner and geographic location as Experiments 1 and 2. This study was planned and conducted prior to learning about the findings of Magid et al., (2018), and prior to Experiment 2. We present it third for ease of interpretation given the stronger methodological overlap between Experiments 1 and 2. Our target sample size was again 20 children per condition per age group (which given the within-subject design meant 80 children). Two additional children were excluded from the analyses for not competing the study.

Materials and Procedures. As in Experiment 2, children began the study with 30 trials of the Number Game to orient them to the task (the impossible trials were not needed to convince children that they weren't perfect, and so were not used here). Then, children were asked to divide the same 14 pairs of questions between two partners as in Experiment 2.

Rather than asking children to divide questions between themselves and a virtual partner, children divided questions between two other children who were playing the Number Game together on a team later that day. The two other children were gender-matched to the participant and presented using small laminated photos. Photos were always placed one above the other rather than beside each other to avoid children associating the partners with sides of the screen.

To introduce the collaborative task, the experimenter said that both partners needed to get all the questions correct to win. One partner was said to be 'good' at the game, while the other partner was said to be 'not so good' at the game. Children were asked a comprehension question about whether each partner was good at the game or not and were corrected if necessary. We asked children to help the partners win the game by splitting up the questions so they could both get them all correct. As in Experiments 1 and 2, children saw pairs of Number Game questions on the screen and selected one question to give to each part- ner. Following the 14 test trials, the experimenter repeated the comprehension questions to check if children remembered the skill difference.

\section{Results}

There was once again no impact of children's gender or metaratio on the results, so these variables were removed from further analyses. Twelve children failed the post-test comprehension check (i.e., incorrectly identified the skill of both partners), but the results below do not change when removing these children. We also confirmed that children at all ages understood the Number Game and selected the more numerous color during the 30 warm-up trials (see Table 3 ).

Table 3

Means and Tests Against Chance in Experiment 3

\begin{tabular}{|c|c|c|c|c|c|c|}
\hline $\begin{array}{l}\text { Age } \\
\text { (Years) }\end{array}$ & Mean & $\mathrm{SD}$ & $\mathrm{df}$ & $t$ & $p$ & $d$ \\
\hline \multicolumn{7}{|c|}{ Number Game (chose more numerous) } \\
\hline 4 & 62.17 & 17.07 & 19 & 3.19 & .005 & 0.71 \\
\hline 5 & 79.00 & 11.75 & 19 & 11.03 & $<.001$ & 2.47 \\
\hline 6 & 82.67 & 7.84 & 19 & 18.63 & $<.001$ & 4.16 \\
\hline 7 & 88.17 & 4.39 & 19 & 38.88 & $<.001$ & 8.69 \\
\hline \multicolumn{7}{|c|}{ Division (gave easier to unskilled) } \\
\hline 4 & 52.14 & 13.73 & 19 & 0.70 & .494 & 0.16 \\
\hline 5 & 63.93 & 25.54 & 19 & 2.44 & .025 & 0.55 \\
\hline 6 & 67.50 & 18.02 & 19 & 4.34 & $<.001$ & 0.97 \\
\hline 7 & 72.50 & 21.40 & 19 & 4.70 & $<.001$ & 1.05 \\
\hline
\end{tabular}

As this study was within-subjects, we were most interested to know if children were assigning harder questions to the 'good' partner and therefore easier questions to the 'not so good' partner. As shown in Table 3 and Figure 4, children aged 5 and older followed this pattern significantly above chance of $50 \%$. A oneway ANOVA found that children were significantly more likely to match difficulty with skill at older age groups, $F(3,76)=3.70, p=.015, \eta_{\mathrm{p}}{ }^{2}=.13$. Specifically, 7-year-olds were significantly more likely to match difficulty and skill than were 4-year-olds, Tukey posthoc $t(38)=20.36, p=.012, d=1.13$. Thus, when children's self-interest was removed by using a third-party task rather than a first-person task, children as young as 5 years old demonstrated strategic behavior consistent with the law of comparative advantage - assigning harder questions to the most competent partner. We repeat this analysis treating age continuously and only using performance on the first trial, and find that these 
results are robust to these modifications (see Supplemental Material).

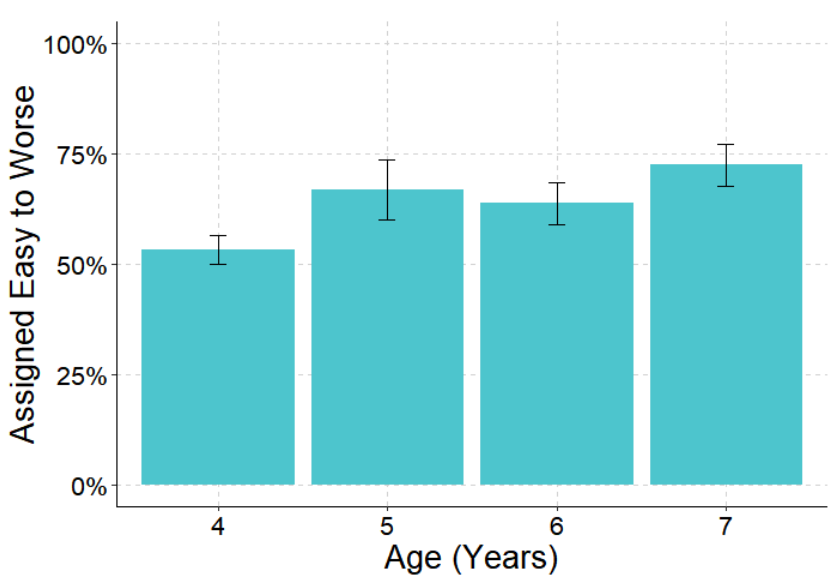

Figure 4. Percentage of Trials Assigning Easier Questions to Worse Partner in Experiment 3. Error bars represent 1 standard error.

\section{General Discussion}

It can be challenging to optimally allocate tasks to group members with differing skills. In three studies, we examined whether children from ages 4-9 apply a strategy based on the law of comparative advantage to their collaborations in a numerical discrimination task. In Experiment 1, children aged 6-9 assigned themselves easier questions when paired with a higherskilled partner, consistent with the law of comparative advantage. But surprisingly, only 9 -year-olds consistently assigned themselves the harder questions if their partner was less skilled. In Experiment 2, 7-year-olds began to demonstrate this strategic reasoning when their partner was described as older or younger, suggesting that at least some of the apparent failure in Experiment 1 might stem from children's developing ability to estimate the probability of their partner's success. In Experiment 3, 5-7-year-olds assigned harder questions to a child 'good' at the task and easier questions to a child 'not so good' at the task in a third-party paradigm where children were not direct participants in the collaboration. This suggests that another influence on performance in Experiment 1 was likely a self-serving bias, in which children knew the best split of questions, but did not enact it as a strategy out of a desire to privilege themselves.

These findings paint a nuanced picture of developing labor division. First, they demonstrate that by 5 years, children can enact a collaborative strategy in the domain of cognitive labor consistent with the law of comparative advantage, in which a higher-skilled partner completes difficult tasks given the greater advantage to the team. This extends prior work of strategic collaboration on a physical task (throwing objects; Magid et al., 2018) and demonstrates breadth of this reasoning to cognitive tasks (reasoning about numbers). Second, they show that enacting this strategy can be constrained by a self-serving bias. Despite understanding the collaborative strategy at age 5, children didn't enact this strategy in their own collaborations until 7-9 years. Like findings in the literature on children's fairness, developing collaborations appear to also be influenced by goals to benefit the self above others. Third, at least some of the effects can be explained by how ability was communicated to the children. Seven-year-old children did not enact a strategy consistent with the law of comparative advantage when their partner was "not as good" as them, but did when their partner was described as "younger". This highlights a caution for researchers interested in measuring or manipulating beliefs about ability or competence: we may underestimate children's reasoning by using abstract terms like "better".

\section{Strategizing About Skill on Cognitive Tasks}

Our goal was to examine the breadth of children's collaborative strategies, and to test what components of their strategy use might differ when children apply the same strategy in different domains. To that end, our results provide several new insights and highlight new directions for understanding how children strategize about cognitive skill.

With respect to the way children estimate the chances of success on cognitive tasks, these findings demonstrate that certain cues are more tightly linked to judgments of skill than others. A simple change in the partner's skill manipulation from an abstract comparison ('better') to an age comparison ('older') resulted in children applying the law of comparative advantage at age 7 rather than 9. This could signal that children begin to learn about skills by tracking the co-occurrence between performance and observable traits, later mapping that understanding onto abstract comparisons. At the same time, children as young as 5 years interpreted the abstract term 'good' to mean skilled in Experiment 3. A fruitful avenue for future research will be to uncover how children learn which cues are relevant to their judgments of skill, and what other cues children use (e.g., stereotypes about group membership; Bian et al., 2017, or observed performance; Ronfard \& Corriveau, 2016). 
With respect to formulating a strategy consistent with the law of comparative advantage, we replicate and extend the findings of Magid and colleagues (2018) that this strategy emerges early in childhood. We found success at 5 years, but not at 4 years as in their study. However, we do not want to make strong claims that the strategy must be learned independently for the physical and cognitive domains based on this small difference. The number task we used has recently been used in studies about children's solo strategies, but with limited success in children under age 5 (e.g., Baer \& Odic, 2019). In contrast, 4-year-olds applied the same strategies on area discrimination (identifying the larger of two shapes), a dimension that shows earlier and more rapid development than number (Baer et al., 2021; Odic, 2018). Therefore, the apparent failure of 4year-olds in Experiment 3 may have more to do with children's difficulty discriminating the difficulties rather than not understanding the strategy. Future work with different tasks or more disparate difficulties that 4-year-olds can reliably detect will be necessary to pin down when this strategy emerges.

A second avenue for future exploration in strategy formation is what computations children perform when devising this strategy. The formal computations for the law of comparative advantage involve estimating four separate probabilities of success and then comparing their subtractions. Could children accomplish the same outcome without these formal calculations? A simple strategy could be to pair easier questions with the less skilled group member, without thinking about the alternative of giving those easier questions to the skilled partner. With this approach, children could theoretically formulate a strategy prior to estimating each party's chances of success on each individual trial (e.g., by deciding to give all easy questions to the partner upon hearing they are not skilled, before seeing any questions to be divided). This could explain children's success in the third-party task, where matching skill to difficulty results in the optimal strategy. However, we would have expected to see similar success in Experiments 1 and 2 if this were true, yet we did not. One means of testing this alternative is to present children with a situation in which the law of comparative advantage favors the opposite pattern: assigning the harder items to the unskilled partner. This would happen when both items are very difficult, making the small increased chances of success by the skilled partner on the easier question comparatively more advantageous for the group.

Our results also prompt future exploration into the use of this strategy in other cognitive skills and other domains of labor. We focused here on a well-studied perceptual magnitude task, but it would also be interesting to explore how children divide labor on other cognitive tasks. For memory, do children spend more time trying to remember items that they have unique access to over items that a partner can also access? Or for language, do bilingual children consider each party's linguistic skill when negotiating which language to communicate in? Outside of physical and cognitive labor, future work could also explore how children collaborate on emotional labor, such as dividing care for others. The law of comparative advantage theoretically applies to all these tasks, provided there is a way to quantify 'success' in each domain.

With respect to enacting the chosen solution, children in our studies were influenced by a self-serving bias, which could stem from several sources. One possibility is that children were motivated to seek rewards (i.e. positive feedback from answering correctly) after being compared to a peer (Magid \& Schulz, 2015). Another possibility is that children were driven to reduce their cognitive effort, choosing questions that could be answered very quickly and without much thought (see Halberda \& Feigenson, 2008; Niebaum \& Munakata, 2020). Yet another possibility is that children were driven to repair their reputation following an unfavorable comparison (see Shaw et al., 2014 for an example within the literature on fairness). A growing body of work suggests that children are very concerned with maintaining their reputation (Silver \& Shaw, 2018), particularly about competence (Asaba \& Gweon, 2019; Zhao et al., 2018). Preliminary evidence of this possibility comes from Experiment 2, where we found that younger children claimed to be more skilled regardless of how old their partner was, whereas older children matched skill to relative age in both conditions. As no such bias was seen when children collaborated on a physical task (Magid et al., 2018), this may reveal differences in how children value their reputation for competency in different domains. Cognitive competency might be especially tied to one's identity and desired reputation given its reliance on properties of the mind, which is more closely linked to the self than other parts of the body (Starmans \& Bloom, 2012). Proving one's cognitive skill may be particularly important for children as they develop independent identities.

Particularly relevant when considering self-serving bias are the specific properties of our studies which may limit generalization. For instance, children had to take the experimenter's word about a virtual partner, which may have led children to doubt the true collabo- 
rative nature of the task. Without full belief in the collaborative goal, children may have fallen back on a rational solo strategy of always answering the easiest questions (Baer et al., 2018; Baer \& Odic, 2019). Further, we only recruited children from Vancouver, British Columbia, Canada and tested them in school settings, which reflect a very small portion of children in the world (Henrich et al., 2010; Nielsen et al., 2017). Children in other societies or testing locations have shown even stronger self-serving tendencies. One cross-cultural study of fairness across seven societies, for instance, found that only in some societies worldwide (including Canada where testing took place) would children avoid advantageous situations (Blake et al., 2015). We might expect similar cultural differences in collaborative strategies, as well.

\section{Implications and Conclusions}

In conclusion, 5-year-olds enact a collaborative strategy consistent with the law of comparative advantage. Our results suggest that children's performance was somewhat limited by challenges in estimating another's chances of success, and more dramatically influenced by self-serving bias when enacting the strategy.

These findings showcase a basic understanding early in life about how to strategically divide cognitive labor, something we often associate with highly-trained economists and managers yet remains a fundamental part of human collaboration. This contributes to ongoing investigations into the cognition enabling humans to engage in sophisticated social behaviors. For example, our work provides initial evidence that children use their own metacognitive confidence to assess what is likely for both their own success and for others' success. This hypothesis can then be applied to many other social behaviors including teaching, helping, and communicating that all rely on understanding others' capabilities.

Further, knowing that this basic strategic understanding is influenced by self-serving biases can be extremely informative for those interested in developing better strategic thinking. For instance, decision-making might be best when developing a strategy for others rather than for the self (see Kross \& Grossmann, 2012). Our work also highlights how some cues to competency might lead to different attitudes about capability. This could be informative for work on modifying attitudes around people with disabilities, where accommodations for 'visible' disabilities are more positively viewed than for 'invisible' disabilities (e.g., a broken leg vs. dyslexia; Deckoff-Jones \& Duell, 2018). Future work into the factors impacting strategic decisions and their development will help refine training programs for these purposes.

\section{References}

Asaba, M., \& Gweon, H. (2019). Young children can rationally revise and maintain what others think of them. PsyArXiv. https://doi.org/10.31234/osf.io/yxhv5

Baer, C., \& Friedman, O. (2018). Fitting the message to the listener: Children selectively mention general and specific facts. Child Development, 89, 461-475. https://doi.org/10.1111/cdev.12751

Baer, C., Gill, I. K., \& Odic, D. (2018). A domain-general sense of confidence in children. Open Mind: Discoveries in Cognitive Science, 2, 86-96. https://doi.org/10.1162/opmi_a_00020

Baer, C., Malik, P., \& Odic, D. (2021). Are children's judgments of another's accuracy linked to their metacognitive confidence judgments? Metacognition and Learning, 16(2), 485-516. https://doi.org/10.1007/s11409-021-09263-x

Baer, C., \& Odic, D. (2019). Certainty in numerical judgments develops independently of the Approximate Number System. Cognitive Development, 52, https://doi.org/10.1016/j.cogdev.2019.100817

Baer, C., \& Odic, D. (2020). The relationship between children's approximate number certainty and symbolic mathematics. Journal of Numerical Cognition, 6(1), 50-65. https://doi.org/10.5964/jnc.v6i1.220

Balcomb, F. K., \& Gerken, L. (2008). Three-year-old children can access their own memory to guide responses on a visual matching task. Developmental Science, 11, 750-760. https://doi.org/10.1111/j.1467-7687.2008.00725.x

Bellon, E., Fias, W., \& Smedt, B. D. (2020). Metacognition across domains: Is the association between arithmetic and metacognitive monitoring domain-specific? PLOS ONE, 15, e0229932. https://doi.org/10.1371/journal.pone.0229932

Bennett-Pierre, G., Asaba, M., \& Gweon, H. (2018). Preschoolers consider expected task difficulty to decide what to do and whom to help. In C. W. Kalish, M. Rau, J. Zhu, \& T. T. Rogers (Eds.), Proceedings of the 40th Annual Meeting of the Cognitive Science Society.

Bian, L., Leslie, S.-J., \& Cimpian, A. (2017). Gender stereotypes about intellectual ability emerge early and influence children's interests. Science, 355, 389-391. https://doi.org/10.1126/science.aah6524

Birch, S. A. J., Vauthier, S. A., \& Bloom, P. (2008). Three-and fouryear-olds spontaneously use others' past performance to guide their learning. Cognition, 107, 1018-1034. https://doi.org/10.1016/j.cognition.2007.12.008

Blake, P. R. (2018). Giving what one should: Explanations for the knowledge-behavior gap for altruistic giving. Current Opinion in Psychology, 20, 1-5. https://doi.org/10.1016/j.copsyc.2017.07.041

Blake, P. R., \& McAuliffe, K. (2011). "I had so much it didn't seem fair": Eight-year-olds reject two forms of inequity. Cognition, 120(2), 215-224. https://doi.org/10.1016/j.cognition.2011.04.006

Blake, P. R., McAuliffe, K., Corbit, J., Callaghan, T. C., Barry, O., Bowie, A., Kleutsch, L., Kramer, K. L., Ross, E., Vongsachang, H., Wrangham, R., \& Warneken, F. (2015). The ontogeny of fairness in seven societies. Nature, 528, 258-261. https://doi.org/10.1038/nature15703 
Blake, P. R., McAuliffe, K., \& Warneken, F. (2014). The developmental origins of fairness: The knowledge-behavior gap. Trends in Cognitive Sciences, 18(11), 559-561. https://doi.org/10.1016/j.tics.2014.08.003

Brainard, D. H. (1997). The Psychophysics Toolbox. Spatial Vision, 10, 433-436. https://doi.org/10.1163/156856897X00357

Bridgers, S., Jara-Ettinger, J., \& Gweon, H. (2020). Young children consider the expected utility of others' learning to decide what to teach. Nature Human Behaviour, 4(2), 144-152. https://doi.org/10.1038/s41562-019-0748-6

Call, J., \& Carpenter, M. (2001). Do apes and children know what they have seen? Animal Cognition, 3, 207-220. https://doi.org/10.1007/s100710100078

Chernyak, N., \& Sobel, D. M. (2015). Equal but not always fair: Value-laden sharing in preschool-aged children. Social Development. http://onlinelibrary.wiley.com.ezproxy.library.ubc.ca/doi/10.1111/sode.12136/pdf

Coughlin, C., Hembacher, E., Lyons, K. E., \& Ghetti, S. (2015). Introspection on uncertainty and judicious help-seeking during the preschool years. Developmental Science, 18, 957-971. https://doi.org/10.1111/desc. 12271

Deckoff-Jones, A., \& Duell, M. N. (2018). Perceptions of appropriateness of accommodations for university students: Does disability type matter? Rehabilitation Psychology, 63(1), 68-76. https://doi.org/10.1037/rep0000213

Geurten, M., Meulemans, T., \& Lemaire, P. (2018). From domainspecific to domain-general? The developmental path of metacognition for strategy selection. Cognitive Development, 48 , 62-81. https://doi.org/10.1016/j.cogdev.2018.08.002

Goupil, L., Romand-Monnier, M., \& Kouider, S. (2016). Infants ask for help when they know they don't know. Proceedings of the National Academy of Sciences, 113, 3492-3496. https://doi.org/10.1073/pnas.1515129113

Halberda, J., \& Feigenson, L. (2008). Developmental change in the acuity of the "Number Sense": The Approximate Number System in 3-, 4-, 5-, and 6-year-olds and adults. Developmental Psychology, 44, 1457-1465. https://doi.org/10.1037/a0012682

Harris, P. L., Koenig, M. A., Corriveau, K. H., \& Jaswal, V. K. (2018). Cognitive foundations of learning from testimony. Annual Review of Psychology, 69, 251-273. https://doi.org/10.1146/annurev-psych-122216-011710

Hembacher, E., \& Ghetti, S. (2014). Don't look at my answer: Subjective uncertainty underlies preschoolers' exclusion of their least accurate memories. Psychological Science, 25, 17681776. https://doi.org/10.1177/0956797614542273

Henrich, J., Heine, S. J., \& Norenzayan, A. (2010). The weirdest people in the world? Behavioral and Brain Sciences, 33(2-3), 61-83. https://doi.org/10.1017/S0140525X0999152X

Koenig, M. A., \& Harris, P. L. (2005). Preschoolers mistrust ignorant and inaccurate speakers. Child Development, 76, 12611277. https://doi.org/10.1111/j.1467-8624.2005.00849.x

Kross, E., \& Grossmann, I. (2012). Boosting wisdom: Distance from the self enhances wise reasoning, attitudes, and behavior. Journal of Experimental Psychology: General, 141(1), 43-48. https://doi.org/10.1037/a0024158

Lutz, D. J., \& Keil, F. C. (2002). Early Understanding of the Division of Cognitive Labor. Child Development, 73(4), 10731084. https://doi.org/10.1111/1467-8624.00458

Lyons, K. E., \& Ghetti, S. (2011). The development of uncertainty monitoring in early childhood. Child Development, 82, 17781787. https://doi.org/10.1111/j.1467-8624.2011.01649.x
Magid, R. W., DePascale, M., \& Schulz, L. E. (2018). Four- and 5year-olds infer differences in relative ability and appropriately allocate roles to achieve cooperative, competitive, and prosocial goals. Open Mind, 2, 72-85. https://doi.org/10.1162/opmi_a_00019

Magid, R. W., \& Schulz, L. E. (2015). Quit while you're ahead: Preschoolers' persistence and willingness to accept challenges are affected by social comparison. Proceedings of the Annual Meeting of the Cognitive Science Society.

Mills, C. M. (2013). Knowing when to doubt: Developing a critical stance when learning from others. Developmental Psychology, 49, 404-418. https://doi.org/10.1037/a0029500

Niebaum, J., \& Munakata, Y. (2020). Deciding What to Do: Developments in Children's Spontaneous Monitoring of Cognitive Demands. Child Development Perspectives, 14(4), 202-207. https://doi.org/10.1111/cdep.12383

Nielsen, M., Haun, D., Kärtner, J., \& Legare, C. H. (2017). The persistent sampling bias in developmental psychology: A call to action. Journal of Experimental Child Psychology, 162, 3138. https://doi.org/10.1016/j.jecp.2017.04.017

Odic, D. (2018). Children's intuitive sense of number develops independently of their perception of area, density, length, and time. Developmental Science, 21, e12533. https://doi.org/10.1111/desc. 12533

Pasquini, E. S., Corriveau, K. H., Koenig, M., \& Harris, P. L. (2007). Preschoolers monitor the relative accuracy of informants. Developmental Psychology, 43(5), 1216.

Pouget, A., Drugowitsch, J., \& Kepecs, A. (2016). Confidence and certainty: Distinct probabilistic quantities for different goals. Nature Neuroscience, 19, 366-374. https://doi.org/10.1038/nn.4240

Ricardo, D. (1891). Principles of Political Economy and Taxation. G. Bell and sons.

Rochat, P., Dias, M. D. G., Liping, G., Broesch, T., Passos-Ferreira, C., Winning, A., \& Berg, B. (2009). Fairness in Distributive Justice by 3- and 5-Year-Olds Across Seven Cultures. Journal of Cross-Cultural Psychology, 40(3), 416-442. https://doi.org/10.1177/0022022109332844

Ronfard, S., \& Corriveau, K. H. (2016). Teaching and preschoolers' ability to infer knowledge from mistakes. Journal of Experimental Child Psychology, 150, 87-98. https://doi.org/10.1007/s13164-017-0343-6

Ronfard, S., \& Harris, P. L. (2017). Children's decision to transmit information is guided by their evaluation of the nature of that information. Review of Philosophy and Psychology, 1-13. https://doi.org/10.1007/s13164-017-0344-5

Shatz, M., \& Gelman, R. (1973). The development of communication skills: Modifications in the speech of young children as a function of listener. Monographs of the Society for Research in Child Development, 38, 1-38. https://doi.org/10.2307/1165783

Shaw, A., Montinari, N., Piovesan, M., Olson, K. R., Gino, F., \& Norton, M. I. (2014). Children develop a veil of fairness. Journal of Experimental Psychology: General, 143, 363-375. https://doi.org/10.1037/a0031247

Sheskin, M., Nadal, A., Croom, A., Mayer, T., Nissel, J., \& Bloom, P. (2016). Some Equalities Are More Equal Than Others: Quality Equality Emerges Later Than Numerical Equality. Child Development, $\quad$ 87(5), https://doi.org/10.1111/cdev.12544

Silver, I. M., \& Shaw, A. (2018). Pint-Sized Public Relations: The Development of Reputation Management. Trends in Cognitive Sciences, 22(4), 277-279. https://doi.org/10.1016/j.tics.2018.01.006 
Simmons, J. P., Nelson, L. D., \& Simonsohn, U. (2011). False-Positive Psychology: Undisclosed Flexibility in Data Collection and Analysis Allows Presenting Anything as Significant. Psychological Science, 22, 1359-1366. https://doi.org/10.1177/0956797611417632

Starmans, C., \& Bloom, P. (2012). Windows to the soul: Children and adults see the eyes as the location of the self. Cognition, 123(2), 313-318. https://doi.org/10.1016/j.cognition.2012.02.002

The Math Works Inc. (2015). MATLAB (9.0 (R2015b)) [Computer software]. The Math Works Inc.

Tomasello, M. (1999). The cultural origins of human cognition (pp. vi, 248). Harvard University Press.

Vo, V. A., Li, R., Kornell, N., Pouget, A., \& Cantlon, J. F. (2014). Young children bet on their numerical skills metacognition in the numerical domain. Psychological Science, 25, 1712-1721. https://doi.org/10.1177/0956797614538458

Warneken, F. (2018). How Children Solve the Two Challenges of Cooperation. Annual Review of Psychology, 69(1), 205-229. https://doi.org/10.1146/annurev-psych-122216-011813

Warneken, F., Steinwender, J., Hamann, K., \& Tomasello, M. (2014). Young children's planning in a collaborative problemsolving task. Cognitive Development, 31, 48-58.

Zhao, L., Heyman, G. D., Chen, L., \& Lee, K. (2018). Telling young children they have a reputation for being smart promotes cheating. Developmental Science, 21(3), e12585. https://doi.org/10.1111/desc.12585 